\title{
Reacción acneiforme como efecto adverso del apremilast
}

\section{Acneiform reaction as an adverse effect of apremilast}

\author{
Angélica Trujillo ${ }^{1}$, Mara Flamini², Pamela Díaz ${ }^{3}$, Sandra García $^{4}$ e Ítalo Aloise ${ }^{5}$
}

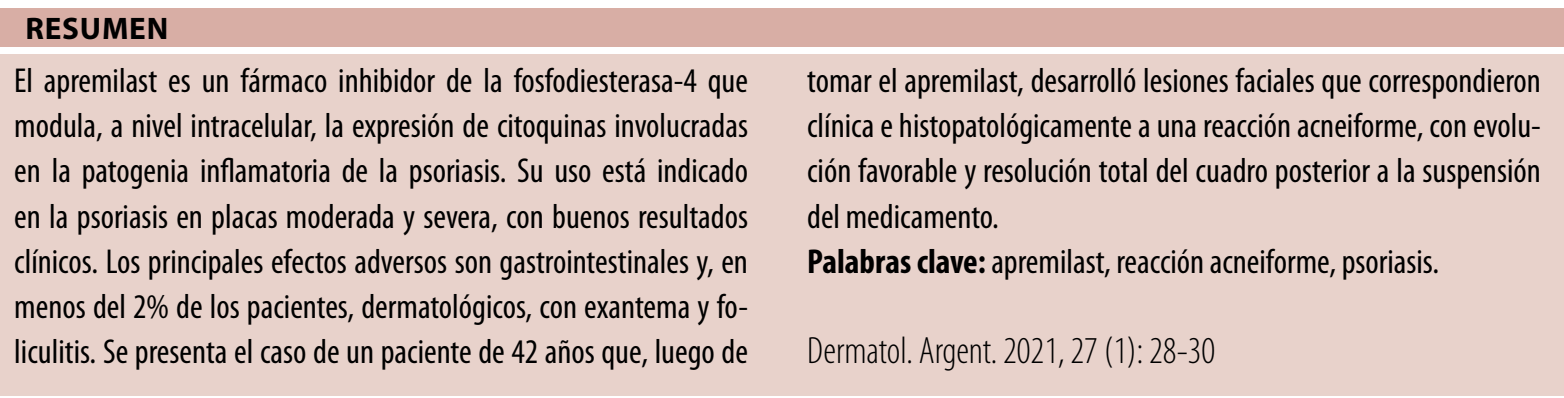

\section{ABSTRACT}

Apremilast is a phosphodiesterase-4 inhibitor that modulates the intracellular expression of cytokines, which are involved in the pathogenesis of psoriasis. Apremilast is indicated in moderate to severe plaque psoriasis, and it has shown good clinical results. The main adverse effects occur at a gastrointestinal level, and in less than $2 \%$ at the dermatologic level with exanthema and folliculitis. We present a 42-year-old patient that developed facial lesions after taking apremilast. The facial lesions were clinically and histopathologically correspond to an acneiform eruption. The patient evolved favorably and fully recovered after suspending apremilast Key words: apremilast, acneiform eruption, psoriasis.

Dermatol. Argent. 2021, 27 (1): 28-30

\author{
1 Médica Residente \\ ${ }^{2}$ Exjefa de Residentes \\ ${ }^{3}$ Médica Dermatóloga, Sector Psoriasis \\ ${ }^{4}$ Dermatopatóloga \\ 5 Jefe del Servicio \\ Servicio de Dermatología, Hospital de Trauma y Emergencias \\ Dr. Federico Abete, Malvinas Argentinas, Provincia de Buenos \\ Aires, Argentina
}

Contacto del autor: Angélica María Trujillo Perdomo E-mail: angelica.trujillo20@gmail.com Fecha de trabajo recibido: 12/2/2021 Fecha de trabajo aceptado: 22/3/2021 Conflicto de interés: los autores declaran que no existe conflicto de interés.

\section{CASO CLÍ́NICO}

Varón de 42 años con antecedentes de hipotiroidismo y psoriasis desde la adolescencia. Realizó diversos tratamientos tópicos, con escasa respuesta. Posteriormente, fue medicado con metotrexato subcutáneo, pero debió suspenderlo por la aparición de hepatotoxicidad y cefalea intensa.

Comenzó el tratamiento con apremilast en dosis de $30 \mathrm{mg}$ cada 12 horas, con reducción del 75\% del Psoriasis Area Severity Index (PASI) basal. Debido a la intolerancia digestiva, a los 3 meses se disminuyó la dosis a la mitad, sin que se produjera rebrote. A los 4 meses de iniciado el tratamiento, se desarrollaron pápulas y pústulas foliculares en la zona de la barba y en la región lateral del cuello, asociadas a gran compromiso de la calidad de vida. No se observaron comedones, eritema centrofacial ni telangiectasias (Fotos 1 y 2). Con la sospecha diagnóstica de reacción acneiforme, foliculitis infecciosa y rosácea, se decidió realizar una biopsia de piel para el estudio microbiológico e histopatológico. El cultivo para gérmenes comunes, micobacterias y hongos resultó negativo. En la microscopia óptica se observó una epidermis conservada y, en la dermis, 
FOTO 1: Pápulas foliculares en la zona de la barba y las mejillas. Ausencia de comedones.

intensos infiltrados inflamatorios mixtos en relación con los anexos pilosebáceos. Los infiltrados incluyeron linfocitos, histiocitos y aisladas células gigantes de tipo cuerpo extraño. No se observaron restos de Demodex folliculorum asociados al proceso inflamatorio ni angiectasias capilares superficiales (Foto 3). Estos hallazgos fueron vinculables a una reacción acneiforme, que se interpretó secundaria al apremilast, por lo que se decidió su interrupción. Se inició tratamiento con minociclina $100 \mathrm{mg} /$ día por vía oral y el paciente retomó el tratamiento con corticosteroides tópicos para el manejo de las lesiones de psoriasis.

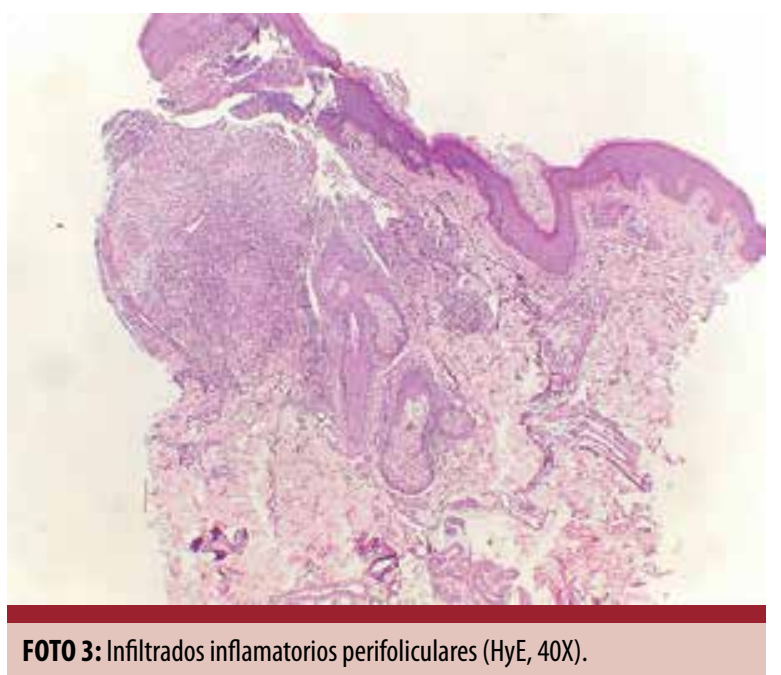

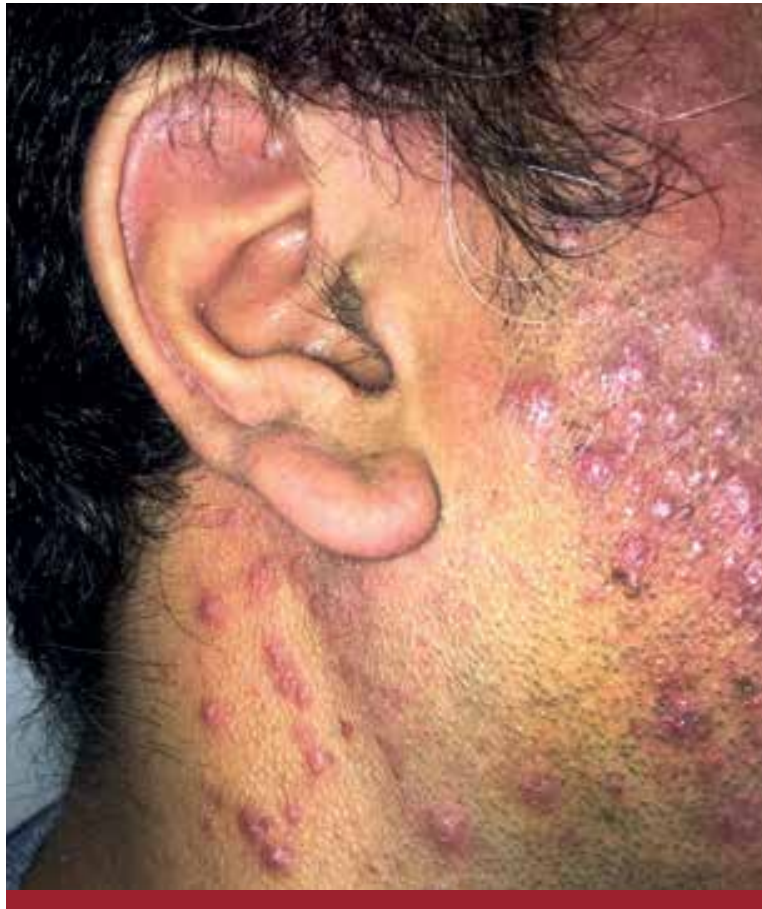

FOTO 2: Pápulas y pústulas foliculares localizadas en la mejilla y la zona lateral del cuello.

A los 2 meses, se observó una marcada mejoría de las lesiones faciales (Foto 4), pero con exacerbación de la psoriasis en la piel y aparición de artritis psoriásica, tras lo cual se inició tratamiento con secukinumab, en dosis de $300 \mathrm{mg}$ mensuales, con excelente respuesta clínica. El paciente no volvió a presentar lesiones acneiformes.

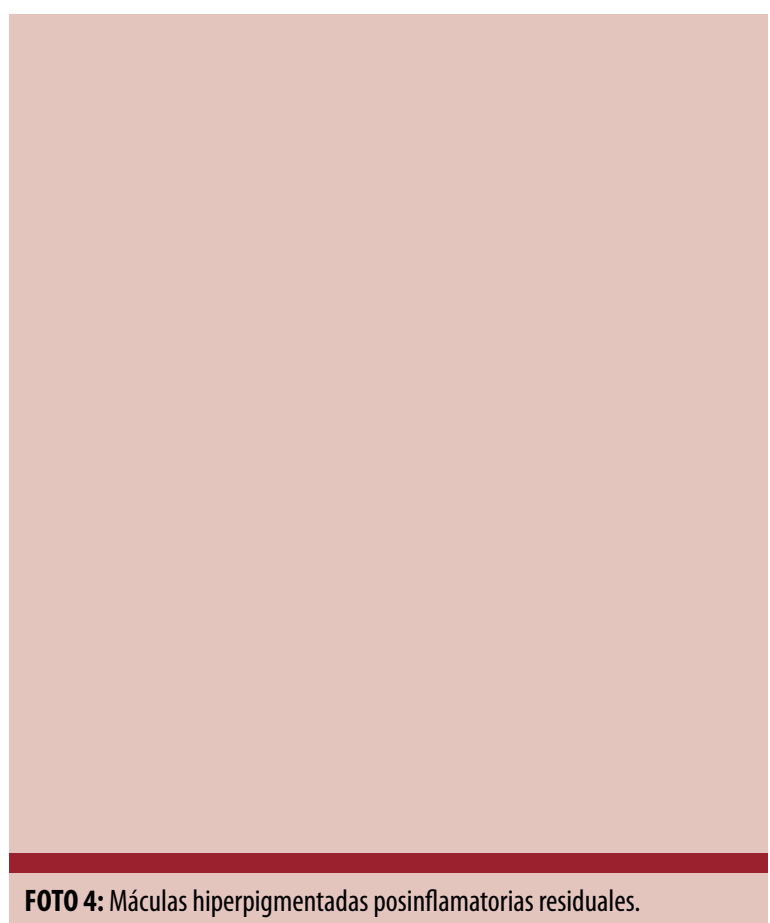




\section{COMENTARIOS}

El apremilast es un inhibidor de la fosfodiesterasa-4 que actúa a nivel intracelular. Aumenta los niveles de AMPc, lo que produce una disminución de la expresión de citoquinas proinflamatorias (TNF-alfa, IL-23, IL-17) y un incremento de los niveles de citoquinas antiinflamatorias como la IL-10 $10^{1}$. Estos mediadores se encuentran involucrados en la patogenia de la psoriasis y la artritis psoriásica. El fármaco está indicado para los pacientes adultos con psoriasis en placas de curso crónico e intensidad moderada a severa que no han respondido a otros tratamientos sistémicos, no los toleran o los tienen contraindicados, como en el caso analizado ${ }^{2,3}$.

$\mathrm{Su}$ uso fue aprobado por la Food and Drug Administation (FDA) en 2014 y en la Argentina a partir de 2018. La dosis recomendada es de $60 \mathrm{mg} / \mathrm{día}^{4}$. Es efectivo en otras variables como prurito, compromiso ungueal, cuero cabelludo y calidad de vida en gene$\mathrm{ral}^{5}$. Sus principales reacciones adversas son de tipo gastrointestinal y se observan en $13-15,7 \%$ de los pacientes. Las manifestaciones más comunes son náuseas, vómitos y diarrea, como ocurrió en el caso clínico comunicado. Menos a menudo, puede provocar infecciones del tracto respiratorio superior y cefalea. Desde el punto de vista dermatológico, se observó una erupción cutánea en menos del $2 \%$ de los pacientes y foliculitis en el $1 \%$ de los $\operatorname{casos}^{6,7}$.

Durante los ensayos clínicos Efficacy and Safety Trial Evaluating the Effects of Apremilast in Psoriasis (ESTEEM) 1 y 2 , el principal motivo de abandono de la medicación fue la diarrea en menos del $2 \%$ de la población estudiada, con una relación dosis-efecto ${ }^{8,9}$.

\section{BIBLIOGRAFÍA}

1. Schafer P. Apremilast mechanism of action and application to psoriasis and psoriatic arthritis. Biochem Pharmacol 2012; 83:1583-1590.

2. Papp K, Reich $\mathrm{K}$, Leonardi $\mathrm{CL}$, Kircik L, et ál. Apremilast, an oral phosphodiesterase 4 (PDE4) inhibitor, in patients with moderate to severe plaque psoriasis: Results of a phase III, randomized, controlled trial (Efficacy and Safety Trial Evaluating the Effects of Apremilast in Psoriasis [ESTEEM] 1). $J$ Am Acad Dermatol 2015;73:37-49.

3. Informe de Posicionamiento Terapéutico de apremilast (Otezla ${ }^{\circ}$ en psoriasis cutánea y artritis psoriásica. Agencia Española de Medicamentos y Productos Sanitarios (AEMPS). 2015 pdf [Internet]. Disponible en: http://www.aemps.gob. es/medicamentosUsoHumano/informesPublicos/docs/IPTapremilast-Otezla.pdf. [Consulta: junio 2020].

4. Del Rosso JQ, Kircik L. Oral Apremilast for the Treatment of Plaque Psoriasis. J Clin Aesthet Dermatol 2016;9:43-48.

5. Sobell JM, Foley P, Toth $D$, Mrowietz U, et ál. Effects of
La reacción acneiforme o dermatitis similar al acné corresponde a una erupción monomorfa, casi siempre localizada en la cara y el cuello, caracterizada por la presencia de lesiones papulopustulosas en ausencia de comedones y en el contexto de un factor desencadenante identificable. Suele asociarse a medicamentos como los corticosteroides tópicos, los esteroides anabólicos como la testosterona, los barbitúricos, el litio, la isoniazida, los antineoplásicos que inhiben el receptor del factor de crecimiento epidérmico (EGFR) y, en menor medida, los derivados de la vitamina $\mathrm{B}$ y los inhibidores del TNF-alfa como infliximab, adalimumab y etanercept. Su etiopatogenia no está bien comprendida hasta el momento. Los hallazgos histopatológicos son variables e incluyen tapones de queratina en infundíbulos foliculares dilatados o rotos, infiltrados inflamatorios perifoliculares y diversos grados de edema. El tratamiento se basa en el retiro del fármaco desencadenante. Si esto no es posible, o según la gravedad de las lesiones, se pueden considerar los queratolíticos, los antibióticos tópicos o la administración de tetraciclinas por vía oral ${ }^{10}$.

Se presenta un caso de reacción acneiforme relacionada con el uso del apremilast, con gran repercusión en la calidad de vida, que motivó la suspensión del fármaco. Si bien esta medicación es una opción de tratamiento en los pacientes que no son candidatos a la terapia sistémica convencional, su uso en nuestro país es relativamente reciente y hace falta más experiencia para conocer mejor sus posibles complicaciones.

Apremilast on Pruritus and Skin Discomfort/Pain Correlate With Improvements in Quality of Life in Patients With Moderate to Severe Plaque Psoriasis. Acta Derm Venereol 2016;96:514-520.

6. Highlights of prescribing information otezla_pi_english.pdf 2020 [Internet]. Disponible en: https://www.pi.amgen.com/ / media/amgen/repositorysites/pi-amgen-com/otezla/otezla_ pi_english.ashx [Consulta: junio 2020].

7. Langley A, Beecker J. Management of common side effects of apremilast. J Cutan Med Surg 2018;22:415-421.

8. Tello E, Suaréz J, Beltrán E, Maldonado C, et ál. Manejo de los efectos adversos de apremilast desde un abordaje multidisciplinar. Actas Dermosifiliogr 2021;112:134-141.

9. Paradela S, Fernández-Torres R, Martínez-Gómez W, Fonseca E. Apremilast en dermatología. Piel 2016;31:206-215.

10. Kazandjieva J, Tsankov N. Drug-induced acne. Clin Dermatol 2017;35:156-162. 